\title{
Invarianza Factorial de la Escala de Ansiedad ante el Envejecimiento de Lasher y Faulkender en Estudiantes Universitarios, Hombres y Mujeres
}

\author{
Susana I. Aguirre, Martha Ornelas, Gabriel Gastélum y Jesús E. Peinado* \\ Universidad Autónoma de Chihuahua. Facultad de Ciencias de la Cultura Física. DES Salud CAs 101 y \\ 121. Calle Escorza 900, CP 31000 Chihuahua, Chih.-México. (e-mail: siaguirre@uach.mx, \\ mornelas@uach.mx,gastelum@uach.mx y jpeinad@uach.mx) \\ ${ }^{*}$ Autor a quien debe ser dirigida la correspondencia.
}

Recibido May. 16, 2016; Aceptado Jul. 20, 2016; Versión final Sep. 14, 2016, Publicado Feb. 2017

\begin{abstract}
Resumen
El presente estudio analiza las propiedades psicométricas de la Escala de Ansiedad ante el Envejecimiento en hombres y mujeres universitarios. La muestra total fue de 1703 participantes; 878 mujeres y 825 hombres, con una edad media de 20.5 años y 20.9 años, respectivamente. Los análisis factoriales confirmatorios mostraron que una estructura tetrafactorial (miedo a las personas mayores, apariencia física, miedo a las pérdidas, y preocupaciones psicológicas) es viable y adecuada para ambas poblaciones (mujeres y hombres) de acuerdo a los requisitos psicométricos establecidos cuando los informantes son los propios alumnos. Además, la estructura factorial, las cargas factoriales y los interceptos se consideran invariantes en las dos poblaciones. Sin embargo, existen diferencias entre las poblaciones para las medias de los factores miedo a las personas mayores y miedo a las pérdidas.
\end{abstract}

Palabras clave: ansiedad; estructura factorial; invarianza de medida; análisis factorial confirmatorio multimuestra

\section{Factorial Invariance of the Anxiety Aging Scale of Lasher and Faulkender of University Students, Men and Women}

\begin{abstract}
The present study analyses the psychometric properties of the Anxiety Aging Scale in men and women university students. The overall sample consisted of 1703 participants: 878 women and 825 men, with a mean age of 20.5 years old and 20.9 years old, respectively. Psychometric analysis showed that a tetrafactorial structure (fear of old people, physical appearance, fear of losses, and psychological concerns) is feasible and adequate for both populations (women and men) according to the established psychometric requirements when the informers are the students themselves. In addition, factor structure, factor loadings and intercepts of the instrument could be considered invariant across groups. However, there are differences between groups for the means of the factors fear of old people and fear of losses.
\end{abstract}

Keywords: anxiety; factor structure; measurement invariance; multigroup confirmatory factor analysis 


\section{INTRODUCCIÓN}

México es un país con una población que envejece rápidamente; a medida que la población de adultos mayores sigue aumentando, también se hace presente la discriminación por edad como la forma más común de prejuicios, fomentando conductas discriminatorias. Para algunas personas la interacción con adultos mayores puede ocasionar ansiedad y tensión e incluso temor, esto podría deberse a la impotencia experimentada sobre el propio futuro en relación con el proceso de envejecimiento, trayendo como consecuencia el aumento de las actitudes discriminatorias contra los ancianos (Bergman y Bodner, 2015).

Tales actitudes negativas hacia el envejecimiento y hacia las personas mayores pueden ser el resultado de una combinación de factores educativos, sociales y económicos, así como la predilección de la cultura consumista orientada a la juventud (Luo et al., 2013). La ansiedad hacia los adultos mayores puede deberse a la falta de conocimiento sobre el proceso del envejecimiento y a la falta de interacción con las personas mayores.

El proceso de envejecimiento tiende a estar asociado con una mayor susceptibilidad a la enfermedad, el deterioro de las funciones corporales y la muerte, relacionando la mala salud con mayor ansiedad ante el envejecimiento conduciendo a actitudes discriminatorias (Brunton y Scott, 2015). Algunas investigaciones sugieren que debido al desconocimiento se tiene una visión negativa sobre el proceso del envejecimiento y muchas personas tienen puntos de vista ageístas hacia los adultos de edad avanzada (Boswell, 2012); mientras que autores como (Allan et al., 2014; Bousfield y Hutchison, 2010) indican que las interacciones voluntarias con los adultos mayores que impliquen cooperación disminuyen la ansiedad, reduce las actitudes discriminatorias y al ser más conscientes de los adultos mayores se experimenta menor preocupación por el propio envejecimiento; mientras que los niveles más altos de ansiedad por el propio envejecimiento parecen contribuir al aumento de las actitudes discriminatorias contra los ancianos.

Los estudios han demostrado consistentemente que son los adultos jóvenes quienes tienen una opinión más negativa y mayor ansiedad acerca del propio envejecimiento que los mayores, y las mujeres las que muestran mayor ansiedad en relación a la apariencia fisca, (Luo et al., 2013; Yan et al., 2011); mientras que los adultos mayores sin distinción de género reportan mayor ansiedad en lo referente a las perdidas (Gao, 2012). Para K. Lasher y P. Faulkender (1993) la ansiedad ante el envejecimiento es un factor importante en la medición de las actitudes y comportamientos hacia las personas de edad avanzada, así como un factor mediador en la adaptación al propio proceso de envejecimiento; estos autores han definido la ansiedad sobre el envejecimiento como la preocupación y la previsión de pérdidas físicas, mentales y personales adversas durante el proceso de envejecimiento; explorando los temores del envejecimiento a través de cuatro dimensiones: miedo a las personas mayores, la apariencia física, problemas psicológicos, y temor a las pérdidas. A medida que la población se vuelve cada vez más vieja se hace más importante comprender la discriminación por edad y desarrollar estrategias para disminuir las actitudes y conductas discriminatorias contra los mayores (Allan et al., 2014).

Por otra parte, los trastornos de ansiedad tienen una alta prevalencia durante toda la vida, con efectos perjudiciales sobre el funcionamiento en la vida diaria, la salud y la calidad de vida (Perna et al., 2016); en los adultos mayores es uno de los problemas de salud mental más presentes y debilitantes y ha sido asociada con enfermedades cardiovasculares, derrame cerebral, deterioro cognitivo, incontinencia urinaria, diabetes, trastornos del sueño, falta de vitalidad, mortalidad ,mayor prevalencia de síntomas de depresión y también con la participación en múltiples comportamientos de riesgo para la salud tales como el tabaquismo, el sedentarismo, la mala alimentación, y el abuso de alcohol (Andreescu y Varon, 2015; Estrada et al., 2013; Ramírez y Palacios-Espinosa, 2016).

En esta misma línea, la ansiedad ha sido asociada con disminución en la función cognitiva, disminución de la actividad física, reducción de la memoria verbal y disminución del rendimiento en las funciones ejecutivas, trayendo como resultado la dificultad para completar varias tareas al mismo tiempo, reduciendo la capacidad de participar en funciones sociales (Potvin et al., 2011; Yochima et al., 2013). De modo similar, se encontró, que la percepción de falta de apoyo social y los estereotipos negativos predice significativamente mayor ansiedad hacia el envejecimiento, y que, las personas experimentan ansiedad al notar deterioro en las actividades básicas de la vida diaria y a medida que necesitan depender de los demás (Arroyo y Soto, 2013; Ramírez y Palacios-Espinosa, 2016).

Un gran número de escalas han sido diseñadas para evaluar las actitudes hacia el envejecimiento y hacia las personas de edad, sin embargo, muchas de estas no han tomado en cuenta la naturaleza multidimensional del envejecimiento y carecen de desarrollo empírico (Sargent-Cox et al., 2014). El presente estudio instrumental (Montero y León, 2005) se ha dirigido a proporcionar apoyo empírico a la división factorial propuesta por Ornelas et al. (2016) para la Escala de Ansiedad ante el Envejecimiento de Lasher y Faulkender (1993); lo que se justifica por la importancia de comprobar la estructura factorial de un 
instrumento y la equivalencia psicométrica del mismo en distintos grupos; ya que en el contexto de la comparación intergrupal, es indispensable plantearse la necesidad de llevar a cabo la adaptación de un instrumento de medida psicológica que cumpla con todos los criterios de equivalencia, pero sobre todo plantearse si la misma estructura factorial es aplicable a distintos grupos de sujetos o, de modo más genérico, a distintas poblaciones (Abalo et al., 2006; Arbuckle, 2012).

Este trabajo pretende, por una parte, pretende indagar si se replican los resultados psicométricos propuestos por Ornelas et al. (2016) para la Escala de Ansiedad ante el Envejecimiento de Lasher y Faulkender (EAELF) y, por otra, ampliarlos. Para ello, en primer lugar se comprobará el grado de congruencia de la estructura factorial del EAELF obtenida en el presente estudio y la reportada por Ornelas et al. (2016). En segundo lugar, se calcula la invarianza factorial entre las muestras del presente estudio.

\section{METODOLOGÍA}

Se describen los participantes, el instrumento de obtención de información, el procedimiento usado y el análisis de los datos recolectados.

\section{Participantes}

La muestra de 1703 participantes, 878 (51.6\%) mujeres y 825 (48.4\%) hombres, se obtuvo mediante un muestreo por conveniencia, tratando de abarcar la representatividad de las diferentes licenciaturas que se ofrecen en la Facultad de Ciencias de la Cultura Física la Universidad Autónoma de Chihuahua. Las edades de las mujeres fluctúan entre los 18 y 28 años, con una media de 20.52 y una desviación estándar de 1.82 años; y las de los hombres fluctúan entre los 18 y 28 años, con una media de 20.90 y una desviación estándar de 2.03 años.

\section{Instrumento}

Escala de Ansiedad ante el Envejecimiento de Lasher y Faulkender versión en español adaptada por Ornelas et al. (2016). Cuestionario tipo Likert, asistida por computadora, que consta de 14 ítems que se agrupan en cuatro dimensiones o subescalas: (1) miedo a las personas mayores, mide el contacto externo con los demás, compuesta por cinco ítems con afirmaciones como "me siento muy a gusto cuando estoy cerca de una persona vieja", (2) apariencia física compuesta por cuatro ítems contiene elementos relacionados con ansiedad acerca de los cambios en la apariencia física, por ejemplo "me ha preocupado el día en que al verme en el espejo me vea cabellos grises", (3) miedo a las pérdidas se refiere a el temor a las pérdidas de apoyo social y la autonomía, compuesta por tres ítems y (4) preocupaciones psicológicas referente a temas personales o internos; compuesta por dos ítems, de acuerdo a Ornelas et al. (2016) las cuatro subescalas tienen una consistencia interna adecuada con alfas de Cronbach por encima de .70.

El encuestado responde, en una escala de 0 a 10 su grado de acuerdo con cada uno de los aspectos propuestos. Las puntuaciones más altas indican mayores niveles de ansiedad ante el envejecimiento.

\section{Procedimiento}

Se invitó a participar en el estudio a los estudiantes de las licenciaturas que se ofrecen en la Facultad de Ciencias de la Cultura Física (FCCF) de la Universidad Autónoma de Chihuahua. Los que aceptaron participar firmaron la carta de aceptación correspondiente. Luego se aplicó el instrumento, antes descrito, por medio de una computadora personal (módulo administrador del instrumento del editor de escalas de ejecución típica), en una sesión de aproximadamente 30 minutos; en los laboratorios o centros de cómputo de la FCCF. Al inicio de cada sesión se hizo una pequeña introducción sobre la importancia de la investigación y de cómo acceder al instrumento. Se les solicitó la máxima sinceridad y se les garantizó la confidencialidad de los datos que se obtuvieran. Las instrucciones de cómo responder se encontraban en las primeras pantallas; antes del primer reactivo del instrumento. Al término de la sesión se les agradeció su participación. Una vez aplicado el instrumento se procedió a recopilar los resultados por medio del módulo generador de resultados del editor de escalas versión 2.0 (Blanco et al., 2013). Una vez aplicado el instrumento se procedió a recopilar los resultados por medio del módulo generador de resultados del editor de escalas versión 2.0 (Blanco et al., 2013).

\section{Análisis de datos}

El análisis psicométrico se realizó en dos etapas: 1) análisis factorial confirmatorio y 2) análisis de invarianza factorial; con el fin de obtener una prueba que presente las mejores propiedades para la conformación de los puntajes de ansiedad ante el envejecimiento en universitarios mujeres y hombres. 
Para conducir el análisis factorial confirmatorio para cada muestra, se utilizó el software AMOS 21 (Arbuckle, 2012), las varianzas de los términos de error fueron especificados como parámetros libres, en cada variable latente (factor) se fijó uno de los coeficientes estructurales asociados a uno, para que su escala sea igual a la de una de las variables superficiales (ítems). El método de estimación empleado fue el de Máxima Verosimilitud; siguiendo la recomendación de Thompson (2004), en el sentido de que cuando se emplea análisis factorial confirmatorio se debe corroborar no sólo el ajuste de un modelo teórico sino que es recomendable comparar los índices de ajuste de varios modelos alternativos para seleccionar el mejor.

Para evaluar el ajuste del modelo se emplearon el estadístico Chi-cuadrado, el índice de bondad de ajuste (GFI) y el error cuadrático medio de aproximación (RMSEA) como medidas absolutas de ajuste. El índice de bondad ajustado (AGFI), el Índice Tucker-Lewis (TLI) y el índice de bondad de ajuste comparativo (CFI) como medidas de ajuste incremental. El índice de ajuste normado de parsimonia (PNFI), el índice de calidad de ajuste de parsimonia (PGFI), la razón de Chi-cuadrado sobre los grados de libertad (CMIN/GL) y el Criterio de Información de Akaike (AIC) como medidas de ajuste de parsimonia (Gelabert et al., 2011). Por último, se llevó a cabo un análisis de la invarianza factorial de los modelos de medida obtenidos, siguiendo las recomendaciones de Abalo et al. (2006), y se calculó la fiabilidad de cada una de las dimensiones a través del Alfa de Cronbach y el Coeficiente Omega (Revelle y Zinbarg, 2009).

\section{RESULTADOS}

Se presentan los resultados en tres subsecciones: (i) análisis factorial confirmatorio; (ii) invarianza de la estructura factorial entre mujeres y hombres universitarios; y (iii) contrastes de las medias de los factores entre mujeres y hombres

\section{Análisis factorial confirmatorio}

De acuerdo a los resultados de la Tabla 1 el análisis factorial confirmatorio de 14 ítems agrupados en cuatro factores en la muestra de mujeres es óptimo (GFI .972 y RMSEA .043) y de acuerdo a las medidas de ajuste incremental y de parsimonia significativamente superior al modelo independiente y muy similar al modelo saturado. Por otro lado, el análisis factorial confirmatorio en la muestra de hombres (Tabla 1), indica que el modelo de medición de cuatro factores es también óptimo (GFI .968 y RMSEA .045) y de acuerdo a las medidas de ajuste incremental y de parsimonia significativamente superior al modelo independiente y muy similar al modelo saturado. En la Tabla 1, ${ }^{*} \mathrm{p}<.05$; GFI = goodness of fit index; RMSEA = root mean square error of approximation; AGFI = adjusted goodness of fit index; TLI = Tucker-Lewis index; $\mathrm{CFI}=$ comparative fit index; CMIN/DF = chi-squared fit index divided by degrees of freedom; AIC = Akaike information criterion.

Tabla 1: Índices absolutos, incrementales y de parsimonia para los modelos generados. Análisis factorial confirmatorio para mujeres y hombres.

\begin{tabular}{|c|c|c|c|c|c|c|c|c|}
\hline \multirow[b]{2}{*}{ Modelo } & \multicolumn{3}{|c|}{ Índices absolutos } & \multicolumn{3}{|c|}{ Índices incrementales } & \multicolumn{2}{|c|}{ Índices de parsimonia } \\
\hline & $\chi^{2}$ & GFI & RMSEA & AGFI & TLI & $\mathrm{CFI}$ & CMIN/DF & AIC \\
\hline \multicolumn{9}{|c|}{ Solución factorial para las mujeres } \\
\hline Independiente & $6104.958^{*}$ & .358 & .275 & .259 & .000 & .000 & 67.087 & 6132.958 \\
\hline Saturado & 0.000 & 1.000 & & & & 1.000 & & 210.000 \\
\hline 4 factores & $183.125^{\star}$ & .972 & .043 & .958 & .976 & .981 & 2.616 & 253.125 \\
\hline \multicolumn{9}{|c|}{ Solución factorial para los hombres } \\
\hline Independiente & $5117.910^{*}$ & .373 & .259 & .277 & .000 & .000 & 56.241 & 5145.910 \\
\hline Saturado & 0.000 & 1.000 & & & & 1.000 & & 210.00 \\
\hline 4 factores & $187.561^{*}$ & .968 & .045 & .953 & .970 & .977 & 2.679 & 257.561 \\
\hline
\end{tabular}

De acuerdo a los resultados de la Tabla 2, en ambas muestras, la mayoría de los ítems saturan adecuadamente en su dimensión prevista. Observándose intercorrelaciones de bajas a moderadas entre los factores evidenciando una buena validez discriminante.

\section{Invarianza de la estructura factorial entre mujeres y hombres universitarios}

Los índices de ajuste obtenidos (Tabla 3) permiten aceptar la equivalencia de los modelos de medida básicos entre las dos muestras. Aunque el valor de Chi-cuadrado excede al exigido para aceptar la hipótesis de invarianza, el resto de índices contradicen esta conclusión (GFI .970; CFI .979; RMSEA .031; AIC 510.687) lo que nos permite aceptar el modelo base de la invarianza (modelo sin restricciones). 
Añadiendo al modelo base restricciones sobre las cargas factoriales caracterizamos la invarianza métrica. Los valores que se recogen en la tabla 3 permiten aceptar este nivel de invarianza. El índice de ajuste general $(\mathrm{GFI}=.970)$ y el error cuadrático medio de aproximación $(\mathrm{RMSEA}=.030)$ siguen aportando información convergente en esta dirección. Además, el criterio de información de Akaike $(A I C=498.667)$ y el índice comparativo de Bentler $(\mathrm{CFI}=.978)$ no sufren grandes variaciones respecto al modelo anterior. Haciendo uso del criterio para la evaluación de los modelos anidados propuesto por Cheung y Rensvold (2002), quiénes sugieren que si el cálculo de la diferencia de los CFI de ambos modelos anidados disminuye en .01 o menos, se da por bueno el modelo restringido y por tanto el cumplimiento de la invarianza factorial; la diferencia entre CFIs obtenida (.001) permite aceptar el modelo de invarianza métrica. Podemos concluir hasta ahora que las cargas factoriales son equivalentes en las dos muestras.

Tabla 2: Soluciones estandarizadas para el análisis factorial confirmatorio en ambas muestras. Nota: F1=Miedo a las personas mayores, F2= Preocupaciones psicológicas, F3=Apariencia física, F4=Miedo a las pérdida

\begin{tabular}{|c|c|c|c|c|c|c|c|c|}
\hline \multirow[b]{2}{*}{ Item } & \multicolumn{4}{|c|}{ Mujeres } & \multicolumn{4}{|c|}{ Hombres } \\
\hline & $\mathrm{F} 1$ & $\mathrm{~F} 2$ & F3 & $\mathrm{F} 4$ & $\mathrm{~F} 1$ & $\mathrm{~F} 2$ & F3 & $\mathrm{F} 4$ \\
\hline \multicolumn{9}{|c|}{ Pesos Factoriales } \\
\hline 1 Disfruto el estar con gente vieja & .81 & & & & .71 & & & \\
\hline 3 Me gusta visitar a mis familiares viejos & 67 & & & & .64 & & & \\
\hline 10 Disfruto platicar con gente vieja & .84 & & & & .83 & & & \\
\hline $\begin{array}{l}13 \text { Me siento muy a gusto cuando estoy cerca de una } \\
\text { persona vieja }\end{array}$ & .82 & & & & .82 & & & \\
\hline 19 Disfruto hacer cosas por los viejos & .75 & & & & .74 & & & \\
\hline 11 Cuando sea viejo creo que voy a sentirme bien con la vida & & .89 & & & & .84 & & \\
\hline $\begin{array}{l}18 \text { Cuando sea viejo, confío en que me sentiré bien conmigo } \\
\text { mismo }\end{array}$ & & .70 & & & & .65 & & \\
\hline 9 Me molesta imaginarme siendo viejo & & & .76 & & & & .71 & \\
\hline $\begin{array}{l}12 \text { Me ha preocupado el día en que al verme en el espejo me } \\
\text { vea cabellos grises }\end{array}$ & & & .77 & & & & .74 & \\
\hline 15 Verme viejo me ha preocupado & & & .87 & & & & .85 & \\
\hline $\begin{array}{l}20 \text { Cuando me veo en el espejo me molesta ver cómo mi } \\
\text { apariencia ha cambiado con la edad }\end{array}$ & & & .70 & & & & .70 & \\
\hline $\begin{array}{l}8 \text { Me pongo nervioso cuando pienso que alguien tomará } \\
\text { decisiones por mí cuando sea viejo }\end{array}$ & & & & .62 & & & & .61 \\
\hline 14 Me preocupa que la gente me ignore cuando sea viejo & & & & 69 & & & & .71 \\
\hline $\begin{array}{l}17 \text { Me preocupa que la vida pierda sentido para mí cuando } \\
\text { sea viejo }\end{array}$ & & & & .80 & & & & .74 \\
\hline \multicolumn{9}{|c|}{ Correlaciones Factoriales } \\
\hline $\mathrm{F} 1$ & - & & & & - & & & \\
\hline $\mathrm{F} 2$ & .61 & - & & & .66 & - & & \\
\hline F3 & .18 & .45 & - & & .23 & .47 & - & \\
\hline $\mathrm{F} 4$ & .00 & .35 & .82 & - & .00 &. .25 & .83 & - \\
\hline
\end{tabular}

Una vez demostrada la invarianza métrica entre las muestras, pasamos a evaluar la equivalencia entre interceptos (invarianza factorial fuerte). Los índices (Tabla 3) muestran un buen ajuste de este modelo, tanto evaluado de modo independiente como analizándolo respecto a su anidamiento con el modelo de invarianza métrica. La diferencia entre los índices comparativos de Bentler es cero; el índice de ajuste general es .969 y el error cuadrático medio de aproximación es .029. Aceptada la invarianza fuerte, los dos modelos evaluados son equivalentes respecto a los coeficientes factoriales y a los interceptos. En la Tabla 3 , * $p<$ .05; GFI = goodness of fit index; NFI = normed fit index; CFI = comparative fit index; RMSEA = root mean square error of approximation; AIC = Akaike information criterion. Todos los valores de consistencia interna, de los factores obtenidos, se encuentran por encima de .70 en ambas muestras (mujeres y hombres); evidenciando una consistencia interna adecuada para este tipo de subescalas, particularmente si se considera el número reducido de ítems (Tabla 4). 
Tabla 3 Índices de bondad de ajuste de cada uno de los modelos puestos a prueba en la invarianza factorial

\begin{tabular}{lccccccc}
\hline \multicolumn{1}{c}{ Modelo } & \multicolumn{7}{c}{ Índice de Ajuste } \\
\cline { 2 - 8 } & $\chi^{2}$ & gl & GFI & NFI & CFI & RMSEA & AIC \\
\hline Modelo sin restricciones & $370.687^{*}$ & 140 & .970 & .967 & .979 & .031 & 510.7 \\
Invarianza métrica & $378.667^{*}$ & 150 & .970 & .966 & .978 & .030 & 498.7 \\
Invarianza factorial fuerte & $392.891^{*}$ & 159 & .969 & .965 & .978 & .029 & 494.9 \\
\hline
\end{tabular}

Tabla 4 Coeficiente omega y alfa para los factores obtenidos

\begin{tabular}{lcccc}
\hline & \multicolumn{2}{c}{ Mujeres } & \multicolumn{2}{c}{ Hombres } \\
\cline { 2 - 5 } Factor & $\Omega$ & $\alpha$ & $\Omega$ & $\alpha$ \\
\hline Miedo a las personas mayores & .886 & .882 & .865 & .862 \\
Preocupaciones psicológicas & .779 & .771 & .715 & .710 \\
Apariencia física & .859 & .857 & .838 & .838 \\
Miedo a las pérdidas & .748 & .769 & .729 & .749 \\
\hline
\end{tabular}

Contrastes de las medias de los factores entre mujeres y hombres

Una vez comprobada la invarianza factorial, las diferencias entre las medias de los factores de los dos grupos se estimaron tomando como referente la muestra de hombres, fijando en 0 el valor de las medias para dicha muestra y estimando libremente el valor de las medias para la muestra de mujeres. Las restricciones sobre los coeficientes de regresión e interceptos, requeridos para los contrastes entre las medias se realizaron automáticamente mediante el software AMOS 21 (Arbuckle, 2012). Los resultados de las comparaciones entre medias indicaron que los hombres presentan mayor ansiedad en el factor "miedo a las personas mayores" $(-0.685, \mathrm{p}<0.001)$ mientras que en el factor "miedo a las pérdidas" son las mujeres las que presentan mayores niveles de ansiedad $(0.369, \mathrm{p}<0.001)$ y sin diferencias significativas en los otros factores (preocupaciones psicológicas y apariencia física).

\section{DISCUSIÓN}

El objetivo principal del estudio fue investigar si se replican o no los resultados psicométricos propuestos por Ornelas et al. (2016) para la Escala de Ansiedad ante el Envejecimiento (EAELF) de Lasher y Faulkender a través de una muestra de universitarios utilizando el análisis factorial confirmatorio. Para ello, en primer lugar se comprobó el grado de congruencia de la estructura factorial del EAELF obtenida en el presente estudio y la reportada por Ornelas et al. (2016). En segundo lugar, se calculó la invarianza factorial entre las muestras del presente estudio.

Los análisis factoriales confirmatorios realizados en la muestras de hombres y mujeres apoyan una estructura factorial de cuatro factores, donde los factores obtenidos presentan en general saturaciones factoriales estandarizadas satisfactorias, modelo que se corresponde con el obtenidos por Ornelas y colaboradores, sugiriendo además la existencia de fuertes evidencias de invarianza factorial entre hombres y mujeres universitarios. En síntesis, el análisis de las propiedades psicométricas, ha mostrado que una estructura tetrafactorial es viable y apropiada; la estructura de cuatro factores, atendiendo a criterios estadísticos y sustantivos, ha mostrado adecuados indicadores de ajuste, de fiabilidad y de validez.

Sin embargo, cabe señalar que el alcance de estos resultados es limitado y por ello es necesario que en investigaciones futuras se confirme la estructura obtenida, lo cual permitirá contar con evidencia más robusta respecto a la estructura factorial de la escala. Específicamente, debe demostrarse si la invarianza de la estructura de la escala se cumple por edad y nivel socioeconómico entre otras; de tal manera que, se considera que más estudios son necesarios con el fin de corroborar o refutar los datos obtenidos en las investigaciones realizadas hasta el momento. Asimismo, es indispensable comprobar si la escala resulta útil para estudiar la relación entre ansiedad ante el envejecimiento y variables como la resiliencia y el bienestar psicológico percibidos en universitarios. 


\section{CONCLUSIONES}

De los resultados mostrados, de su análisis y de su discusión, y tomando en cuenta que el objetivo principal de este estudio fue el de examinar la estructura factorial y la medición de la invariancia de dicha estructura en alumnas y alumnos universitarios, se pueden obtener las siguientes conclusiones:

1) El Análisis Factorial Confirmatorio, en ambas muestras, indicó que el ajuste de los datos al modelo teórico de 14 ítems agrupados en cuatro factores es óptima. Al mismo tiempo que los cuatro factores así obtenidos presentan en general saturaciones factoriales estandarizadas adecuadas. Por su parte los factores correlacionan entre sí de forma positiva y estadísticamente significativa lo cual muestra que a medida que aumenta la ansiedad percibida en alguno de los factores, también aumenta en los otros.

2) Los cuatro factores obtenidos muestran una consistencia interna adecuada.

3) Conjuntamente con todo lo antes dicho, los resultados del análisis de la invarianza factorial entre las muestras; indican una alta congruencia entre pares de factores. Lo que sugiere la existencia de fuertes evidencias de la validación cruzada de la medida y por tanto de la estabilidad de la estructura, hasta que no se demuestre lo contrario.

4) Las comparaciones entre los grupos reflejaron diferencias significativas, a favor de las mujeres en el factor miedo a las personas mayores y a favor de los hombres en el factor miedo a las pérdidas. Lo que parece indicar que las mujeres se perciben más ansiosas en lo relacionado con aspectos tales como la toma de decisiones o la pérdida del sentido de la vida al ser un adulto mayor, mientras que los hombres lo hacen en relación a la convivencia con gente adulta mayor.

\section{REFERENCIAS}

Abalo J., Lévy J., Rial A. y Varela J., Invarianza factorial con muestras múltiples, En Modelización con Estructuras de Covarianzas en Ciencias Sociales por J. Lévy, pp 259-278 Netbiblo, Madrid, (2006)

Allan L., Johnson J. y Emersona S., The role of individual difference variables in ageism, Personality and Individual Differences, 59, 32-37, (2014)

Andreescu C. y Varon D., New research on anxiety disorders in the elderly and an update on evidencebased treatments, Current Psychiatry Reports, 17(7), 1-7, (2015).

Arbuckle J. R., AMOS users guide version 21.0, Marketing Department, SPSS Incorporated, Chicago, IL, (2012)

Arroyo M. y Soto L., La dimensión emocional del cuidado en la vejez: la mirada de los adultos mayores, Cuadernos de Trabajo Social, 26(2), 337-347, (2013).

Bergman Y. y Bodner E., Ageist attitudes block young adults' ability for compassion toward incapacitated older adults, International Psychogeriatrics, 27(9), 1541-1550 (2015)

Blanco H., Ornelas M., Tristán J. L., Cocca A., Mayorga-Vega D., López-Walle J., et al., Editor for creating and applying computerise surveys, doi: http://dx.doi.org/10.1016/j.sbspro.2013.12.105, Procedia Social and Behavioral Sciences, 106, 935-940, (2013)

Boswell S., "Old people are cranky": Helping professional trainees' knowledge, attitudes, aging anxiety, and interest in working with older adults, Educational Gerontology, 38(11), 733-741, (2012)

Bousfield C. y Hutchison P., Contact, anxiety, and young people's attitudes and behavioral intentions towards the elderly, Educational Gerontology, 36(6), 451-466, (2010)

Brunton R. y Scott G., Do We Fear Ageing? A multidimensional approach to ageing anxiety educational gerontology, Educational Gerontology, 41(11), 786-799, (2015)

Cheung G. W. y Rensvold R. B., Evaluating goodness-of-fit indexes for testing measurement invariance, doi: 10.1207/s15328007SEM0902_5, Structural Equation Modeling, 9(2), 233-255, (2002)

Estrada A., Cardona Arango D., Segura Á. M., Ordoñez J., Osorio J. J. y Chavarriaga L. M., Síntomas depresivos en adultos mayores institucionalizados y factores asociados, Universitas Psychologica, 12(1), 8194, (2013). 
Gao Y.-J., Measurement of aging anxiety in taiwan: an application of a multidimensional item response model, Social Behavior and Personality, 40(4), 557-566, (2012)

Gelabert E., García-Esteve L., Martín-Santos R., Gutiérrez F., Torres A. y Subirà S., Psychometric properties of the Spanish version of the Frost Multidimensional Perfectionism Scale in women, Psicothema, 23(1), 133139, (2011)

Lasher K. P. y Faulkender P. J., Measurement of Aging Anxiety: Development of the Anxiety About Aging Scale, International Journal of Aging and Human Development, 37(4), 247-259, (1993)

Luo B., Zhou K., Jung Jin E., Newman A. y Liang J., Ageism among college students: a comparative study between U.S. and China, Journal of Cross-Cultural Gerontology, 28(1), 49-63, (2013)

Montero I. y León O., Sistema de clasificación del método en los informes de investigación en Psicología, International Journal of Clinical and Health Psychology, 5, 115-127, (2005)

Ornelas M., Gastélum G., López-Walle J. y Rodríguez-Villalobos J. M., Composición Factorial de la Escala de Ansiedad ante el Envejecimiento de Lasher y Faulkender en Estudiantes Universitarios Mexicanos, doi: 0.4067/S0718-50062016000200008, Formación Universitaria, 9(2), 73-80, (2016)

Perna G., Lannone G., Alciati A. y Caldirola D., Are Anxiety Disorders Associated with Accelerated Aging? A Focus on Neuroprogression, Neural Plasticity, 2016, 1-19, (2016).

Potvin O., Forget H., Grenier S., Préville M. y Hudon C., Anxiety, depression, and 1-year incident cognitive impairment in community-dwelling older adults, Journal of the American Geriatrics Society, 59(8), 14211428, (2011).

Ramírez L. y Palacios-Espinosa X., Stereotypes about old age, social support, aging anxiety and evaluations of one's own health, Journal of Social Issues, 72(1), 47-68, (2016).

Revelle W. y Zinbarg R. E., Coefficients alpha, beta, omega and the glb: comments on Sijtsma, doi: 10.1007/s11336-008-9102-z, Psychometrika, 74(1), 145-154, (2009)

Sargent-Cox K., Rippon M. y Burns R., Measuring anxiety about aging across the adult lifespan, International Psychogeriatrics, 26(1), 135-145, (2014)

Thompson B., Exploratory and Confirmatory Factor Analysis. Understanding concepts and applications. , American Psychological Association, Washington, D C, (2004)

Yan T., Silverstein M. y Wilber K., Does race/ethnicity affect aging anxiety in american baby boomers?, Research on Aging, 33(4), 361-378, (2011)

Yochima B., Mueller A. y Segal D., Late life anxiety is associated with decreased memory and executive functioning in community dwelling older adults, Journal of Anxiety Disorders, 27(6), 567- 575, (2013) 